DOI: 10.12731/2227-930X-2020-3-114-122 УДК 656.2-027.45

\title{
МЕТОД ЗАЩИТЫ ВОЗДУШНОГО КОРИДОРА ВЫДЕЛЕННОГО ДЛЯ ДВИЖЕНИЯ БЕСПИЛОТНЫХ ЛЕТАТЕЛЬНЫХ АППАРАТОВ
}

\section{Швецов А.В.}

Беспилотные летательные аппараты, получают все большее практическое применение в современном обществе, в различных приложениях. При этом распространение возможностей предоставляемых технологиями и методами, связанными с беспилотными летательными аппаратами, сдерживается из-за нерешенной проблемы обеспечения безопасности их движения, ограничивающей область их применения.

Цель: разработать метод зашиты выделенного воздушного коридора и реализующее его техническое решение, совместно позволяюших повысить безопасность движения беспилотных летательных аппаратов.

Методы: используются системный анализ, синтез, индукиия и дедукция, сравнение, обобщение и другие научные методы исследования.

Результаты: разработан воздушный тоннель, позволяющий беспилотным летательным аппаратам, защищено передвигаться на опасных участках выделенного воздушного коридора. Воздушный тоннель является техническим решением, реализующим на практике предложенный в работе метод защиты выделенного воздушного коридора.

Область применения результатов: разработанный метод и реализующее его техническое решение совместно позволяют повысить безопасность движения гражданских беспилотных летательных аппаратов вертикального взлета и посадки, и применимы для обеспечения безопасности полетов БПЛА на наземных объектах, например, 
таких как промылиленные объекты и крупныле складские комплексы, он также может быть применен при проектировании систем движения беспилотных летательных аппаратов в городах.

Ключевые слова: беспилотный летательный аппарат; БПЛА; метод; обеспечение безопасности.

\section{METHOD OF PROTECTING THE AIR CORRIDOR DEDICATED FOR TRAFFIC UNMANNED AIRCRAFT}

\section{Shvetsov A.V.}

Unmanned aerial vehicles are getting more and more practical application in modern society, in various applications. At the same time, the spread of the possibilities provided by technologies and methods associated with unmanned aerial vehicles is constrained due to the unresolved problem of ensuring the safety of their movement, which limits the scope of their application.

Purpose: to develop a method for protecting a dedicated air corridor and a technical solution that implements it, jointly improving the safety of unmanned aerial vehicles.

Methodology: systems analysis, synthesis, induction and deduction, comparison, generalization and other scientific research methods are used.

Results: An air tunnel was developed that allows unmanned aerial vehicles to move securely in dangerous sections of the designated air corridor. An air tunnel is a technical solution that implements in practice the method of protecting a dedicated air corridor proposed in the work.

Practical implications: the developed method and the technical solution that implements it together make it possible to increase the traffic safety of civil unmanned aerial vehicles with vertical take-off and landing, and are applicable to ensure the safety of UAV flights at ground-based facilities, for example, such as industrial facilities and large warehouse complexes, it can also be applied in the design of systems for the movement of unmanned aerial vehicles in cities.

Keywords: unmanned aerial vehicle; UAV; method; safety. 


\section{Введение}

Безопасная и эффективная интеграция беспилотных летательных аппаратов (БПЛА) в воздушное движение является одной из основных задач в авиации в первой половине 21-го века [1-6]. БПЛА, включая дистанционно управляемые человеком летательные аппараты и автоматизированные летательные аппараты, управляемые программой, находят применение не только в уже существующих приложениях, таких как картография, безопасность, транспортировка [7-10], но и позволяют создавать принципиально новые, в том числе основанные на использовании искусственного интеллекта.

Но, в настоящий момент, распространение возможностей, предоставляемых технологиями и методами, связанными с беспилотными летательными аппаратами, сдерживается существующими ограничениями по применению БПЛА на определенных объектах [11], в первую очередь, аэропортах (рис. 1). Рассматриваемые ограничения вызваны угрозами безопасности, возникающими при полете БПЛА [12-16].

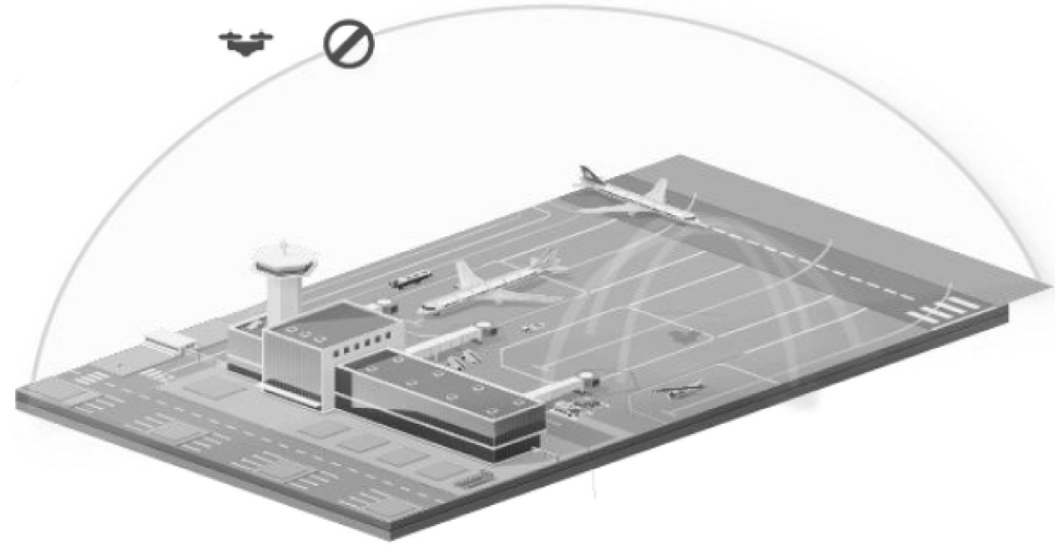

Рис. 1. Геозона над аэропортом, закрытая для движения БПЛА [17]

\section{Метод защиты выделенного воздушного коридора}

Повысить безопасность движения беспилотных летательных аппаратов, возможно за счет обеспечения защищенности границ 
выделенного воздушного коридора, предназначенного для полетов беспилотных летательных аппаратов, на участках, где существует угроза столкновения БПЛА с другими участниками движения (далее - опасные участки), к опасным участкам также необходимо отнести такие, где для беспилотного летательного аппарата существует угроза прямого несанкционированного вмешательства и воздействий со стороны нарушителя, имеющего цель захватить либо повредить БПЛА.

Обеспечение такой защищенности возможно на основе создания сплошного физического барьера между ВВК и внешней средой (рис. 2) на всем протяжении опасного участка.

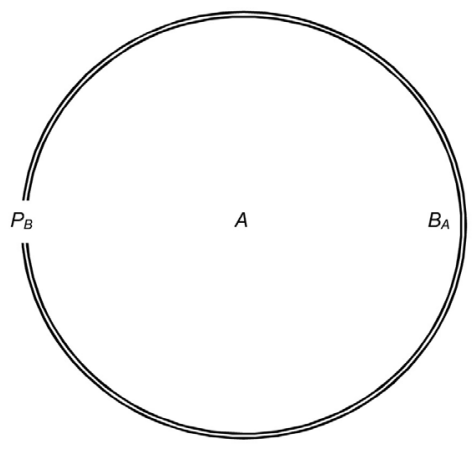

$A-$ ВВК (в разрезе)

$B_{A}-$ границы ВBК;

$P_{B}-$ физический барьер.

Рис. 2. Физический барьер между ВВК и внешней средой

Предложенный метод реализуется техническим решением воздушным тоннелем (ВТ), предназначенным для защищенного прохождения БПЛА опасных участков ВВК.

Разработанный воздушный тоннель позволит БПЛА проходить опасные участки ВВК без угрозы столкновения с другими УД, а также обеспечить защиту беспилотного летательного аппарата от внешнего несанкционированного вмешательства и воздействий.

Детально конструкция воздушного тоннеля показана на рисунке 2. 


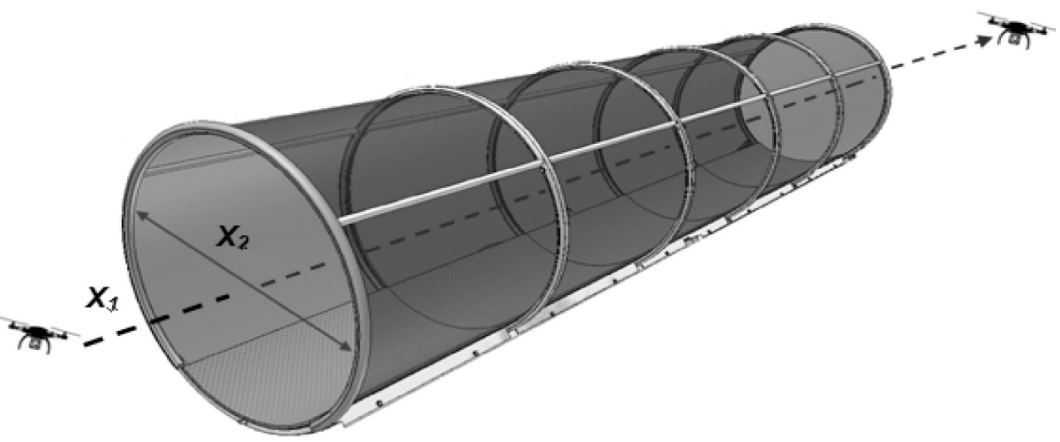

Рис. 2. Воздушный тоннель $\left(\mathrm{X}_{1}-\right.$ траектория полета БПЛА;

$\mathrm{X}_{2}$ - диаметр ВТ (определяется на основании информации о размерах применяемых БПЛА и имеющимся свободном пространстве)

Установка тоннеля в воздушном пространстве ОТИ выполняется с применением дополнительных монтажных металлоконструкций (далее опоры-кронштейны) форма которых зависит от условий монтажа ВТ.

Опоры-кронштейны могут крепиться к специально установленным в грунт винтовым сваям либо к зданиям или сооружениям, расположенным по маршруту движения БПЛА.

При одновременном движении группы БПЛА в воздушном тоннеле их движение может осуществляться только в одном выбранном направлении с безопасным интервалом между ними, это необходимо для предотвращения столкновений БПЛА.

Безопасный интервал $\left(S_{i n t}\right)$ определяется по формуле:

$$
S_{i n t}=T_{\text {res }}+B_{\text {dis }},
$$

где $T_{r e s}-$ дистанция проходимая БПЛА с момента обнаружения оператором препятствия на пути движения БПЛА и до момента применения оператором экстренного торможения;

$B_{d i s}$ - тормозной путь проходимый БПЛА при экстренном торможении.

Формула 1 позволяет рассчитать безопасный интервал для заданной скорости движения БПЛА, для этого в показатели $T_{r e s}$ и $B_{d i s}$ подставляются их значения при рассматриваемой скорости. 
Формула 1 позволяет рассчитать безопасный интервал для заданной скорости движения БПЛА, для этого в показатели $T_{r e s}$ и $B_{d i s}$ подставляются их значения при рассматриваемой скорости.

Ограничения в применении

ВТ применим в приложениях предусматривающих движение БПЛА по определенному маршруту.

ВТ применим на наземных объектах.

ВТ предназначен для движения БПЛА «вертолетного» типа, вертикального взлета и посадки.

\section{Обсуждение}

В настоящей работе авторами предложены метод защиты выделенного воздушного коридора и реализующее его техническое решение. Анализ известных российских и зарубежных источников показывает, что публикации, описывающие аналогичные решения в настоящий момент отсутствуют. Можно сделать вывод, что результаты, представленные в настоящей работе, являются инновационным решением, направленным на повышение безопасности полетов БПЛА, и могут представлять интерес для международного экспертного сообщества при обсуждении путей интеграции беспилотных летательных аппаратов в существующую транспортную систему.

\section{Выводы}

В статье разработаны - метод защиты выделенного воздушного коридора и реализующее его техническое решение - воздушный тоннель.

Воздушный тоннель применим для обеспечения безопасности движения БПЛА на наземных объектах, например, таких как промышленные объекты и крупные складские комплексы и др.

Информация о конфликте интересов. Автор заявляет об отсутствии конфликта интересов.

Информация о спонсорстве. Исследование не имело спонсорской поддержки. 


\section{Список литературы / References}

1. Alsamhi SH, Ma O, Ansari MS. Convergence of Machine Learning and Robotics Communication in Collaborative Assembly: Mobility, Connectivity and Future Perspectives. Journal of Intelligent \& Robotic Systems [Internet]. Springer Science and Business Media LLC; 2019 Oct 16; Available from: http://dx.doi.org/10.1007/s10846-019-01079-x

2. Minucci F, Vinogradov E, Sallouha H, Pollin S. UAV Location Broadcasting with Wi-Fi SSID. 2019 Wireless Days (WD) [Internet]. IEEE; 2019 Apr; Available from: http://dx.doi.org/10.1109/wd.2019.8734208

3. Kanzaki A, Akagi H. A UAV-Collaborative Sensing Method for Efficient Monitoring of Disaster Sites. Advances in Intelligent Systems and Computing [Internet]. Springer International Publishing; 2019 Mar 15;775-86. Available from: http://dx.doi.org/10.1007/978-3-03015032-7_65

4. Jun-yan L, Zhuo-ning D, Meng-yue Z. A fuzzy virtual force based approach to multiple UAVs collaborative path planning. Proceedings of 2014 IEEE Chinese Guidance, Navigation and Control Conference [Internet]. IEEE; 2014 Aug; Available from: http://dx.doi.org/10.1109/ cgncc.2014.7007381

5. Saleem Y, Rehmani MH, Zeadally S. Integration of Cognitive Radio Technology with unmanned aerial vehicles: Issues, opportunities, and future research challenges. Journal of Network and Computer Applications [Internet]. Elsevier BV; 2015 Apr;50:15-31. Available from: http://dx.doi.org/10.1016/j.jnca.2014.12.002

6. Popescu D, Dragana C, Stoican F, Ichim L, Stamatescu G. A Collaborative UAV-WSN Network for Monitoring Large Areas. Sensors [Internet]. MDPI AG; 2018 Nov 30;18(12):4202. Available from: http:// dx.doi.org/10.3390/s18124202

7. Popescu, Stoican, Stamatescu, Chenaru, Ichim. A Survey of Collaborative UAV-WSN Systems for Efficient Monitoring. Sensors [Internet]. MDPI AG; 2019 Oct 28;19(21):4690. Available from: http://dx.doi. org/10.3390/s19214690

8. Schroeder K, Song Y, Horton B, Bayandor J. Investigation of UAS Ingestion into High-Bypass Engines, Part 2: Parametric Drone Study. 58th 
AIAA/ASCE/AHS/ASC Structures, Structural Dynamics, and Materials Conference [Internet]. American Institute of Aeronautics and Astronautics; 2017 Jan 5; Available from: http://dx.doi.org/10.2514/6.2017-0187

9. Shvetsova SV, Shvetsov AV. Safety Analysis of Goods Transportation by Unmanned Aerial Vehicles. World of Transport and Transportation [Internet]. FSBEO HPE Moscow State University of Railway Engineering (MIIT); 2020; 17(5):286-97. Available from: http://dx.doi. org/10.30932/1992-3252-2019-17-5-286-297

10. Alsamhi S, Ma O, Ansari M, Gupta S. Collaboration of Drone and Internet of Public Safety Things in Smart Cities: An Overview of QoS and Network Performance Optimization. Drones [Internet]. MDPI AG; 2019 Jan 27;3(1):13. Available from: http://dx.doi.org/10.3390/ drones3010013

11. Huttunen M. Civil unmanned aircraft systems and security: The European approach. Journal of Transportation Security [Internet]. Springer Science and Business Media LLC; 2019 Sep 23;12(3-4):83-101. Available from: http://dx.doi.org/10.1007/s12198-019-00203-0

12. Sciancalepore S, Ibrahim OA, Oligeri G, Di Pietro R. Detecting Drones Status via Encrypted Traffic Analysis. Proceedings of the ACM Workshop on Wireless Security and Machine Learning - WiseML 2019 [Internet]. ACM Press; 2019; Available from: http://dx.doi. org/10.1145/3324921.3328791

13. Amukele TK, Hernandez J, Snozek CL, Wyatt RG, Douglas M, Amini $\mathrm{R}$, et al. Drone Transport of Chemistry and Hematology Samples Over Long Distances. American Journal of Clinical Pathology [Internet]. Oxford University Press (OUP); 2017 Sep 5;148(5):427-35. Available from: http://dx.doi.org/10.1093/ajcp/aqx090

15. Goodchild A, Toy J. Delivery by drone: An evaluation of unmanned aerial vehicle technology in reducing CO 2 emissions in the delivery service industry. Transportation Research Part D: Transport and Environment [Internet]. Elsevier BV; 2018 Jun;61:58-67. Available from: http://dx.doi.org/10.1016/j.trd.2017.02.017

16. Kirschstein T. Comparison of energy demands of drone-based and ground-based parcel delivery services. Transportation Re- 
search Part D: Transport and Environment [Internet]. Elsevier BV; 2020 Jan;78:102209. Available from: http://dx.doi.org/10.1016/j. trd.2019.102209

17. Airbus'Advanced Inspection Drone. [Internet]. Available from: https:// www.airbus.com/newsroom/press-releases/en/2018/04/airbus-launches-advanced-indoor-inspection-drone-to-reduce-aircr.html

\section{ДАННЫЕ ОБ АВТОРЕ}

Швецов Алексей Владиславович, кандидат технических наук, доцент кафедры транспортных процессов и технологий ВГУЭС; доцент кафедры «Эксплуатация автомобильного транспорта и автосервис» СВФУ

Владивостокский государственный университет экономики и сервиса; Северо-Восточный Федеральный Университет ул. Гоголя, 44, г. Владивосток, 690000, Российская Федераuчия; ул. Белинского, 58, г. Якутск, 677000, Российская Федерачия transport-safety@mail.ru

\section{DATA ABOUT THE AUTHOR}

Shvetsov Alexey Vladislavovich, candidate of technical sciences, Associate Professor of the Department of Transport Processes and Technologies (VSUES); Associate Professor of the Department of Operation of Automobile Transport and Car Service (NEFU) Vladivostok State University of Economics and Service (VSUES); North-Eastern Federal University (NEFU)

44, Gogol Str., Vladivostok, 690000, Russian Federation; 58, Belinsky Str., Yakutsk, 677000, Russian Federation transport-safety@mail.ru ORCID: 0000-0001-5165-2816 